\title{
Estudio del comportamiento hidrodinámico de un filtro biológico de flujo ascendente, utilizando mazorcas de maíz como material de soporte
}

\author{
Study of the hydrodynamic performance of a biological upflow \\ filter, using corn cobs as support material
}

\author{
Madeleine Mollah ${ }^{1}$, Charline Sánchez ${ }^{1}$, Kelsyn Barría ${ }^{1}$, Diamcely Ferrán ${ }^{l}$, Erick Vallester ${ }^{2 *}$ \\ ${ }^{1}$ Facultad de Ingeniería Civil, Universidad Tecnológica de Panamá
}

\begin{abstract}
Resumen Las condiciones de flujo afectan el desempeño de los filtros. Las causas comunes son corto circuitos, zonas muertas y recirculación interna por corrientes internas y/o densidad. El artículo destaca el funcionamiento de un reactor compuesto por un tanque de 114 litros, el cual alimenta de agua cruda a tres filtros biológicos de flujo ascendente, con un volumen de vacío de 8 litros cada uno, que contienen tusas de maíz como material de soporte. Al reactor se le inyectó en el punto de entrada un trazador, aplicándose la técnica de estímulo-respuesta para estudiar su comportamiento hidrodinámico con base a su tiempo de retención. Durante el ensayo de las pruebas se determinaron las curvas de concentración vs tiempo en cada filtro, dando como resultado el tiempo de retención y el volumen real de cada uno. En la evaluación del desempeño del comportamiento hidrodinámico se encontraron variaciones y presencia de flujo dual, como lo son: flujo de pistón y flujo de mezcla completa, evidenciando espacios de zonas muertas, así como también cortos circuitos.
\end{abstract}

Palabras clave Comportamiento hidrodinámico, corto circuito, flujo ascendente, flujo de mezcla completa, flujo pistón, tiempo de retención, trazador.

\begin{abstract}
The flow conditions affect the performance of the filters. The common causes are short circuits, dead zones and internal recirculation by internal currents and / or density. The article highlights the operation of a reactor composed of a tank of 114 liters, which feeds raw water to three upstream biological filters, with a vacuum volume of 8 liters each, which contain corn chips as support material. A tracer was injected at the entry point, applying the stimulus-response technique to study its hydrodynamic performance, based on its retention time. During the process, the concentration vs. time curves in each filter were determined, resulting in the retention time and the actual volume of each one. In the performance evaluation of the hydrodynamic performance, variations and presence of dual flow were found, such as: piston flow and complete mix flow; evidencing spaces of dead zones, as well as short circuits.
\end{abstract}

Keywords Hydrodynamic behavior, short circuit, upflow, complete mixing flow, piston flow, retention time, tracer.

* Corresponding author: erick.vallester@utp.ac.pa

\section{Introducción}

El diseño de los sistemas de tratamiento de aguas residuales se ha centrado fundamentalmente en el proceso bioquímico, dejando en segundo plano aspectos hidrodinámicos como: las características del flujo, el régimen de mezcla, los tiempos de residencia, la geometría del reactor y el estado de agregación de elementos del fluido, los cuales influyen en el desempeño de los procesos que se llevan a cabo durante el tratamiento. Por lo tanto, el conocimiento de la hidrodinámica del sistema permitirá mejorar su eficiencia [1]. El método experimental estimulo-respuesta consiste en inyectar en el afluente un trazador de concentración conocido, detectable y que no participe en ninguno de los procesos físicos y químicos que puedan alterar la hidrodinámica real del fluido. La aplicación del trazador se puede hacer de dos formas: instantánea o continua. La primera consiste en adicionar el trazador de manera rápida en el reactor; esta es la más utilizada. Por otro lado, en el método continuo, el trazador se aplica de modo más rápido y se mantiene constante durante un tiempo para entonces suspenderlo de forma instantánea.

Es una práctica común establecer la eficiencia hidráulica de un reactor analizando la curva de concentración vs tiempo y relacionar los diferentes parámetros con las condiciones del flujo. Generalmente, la forma que toma la curva de 
Mollah (et al): Estudio del comportamiento hidrodinámico de un filtro biológico de flujo ascendente, utilizando mazorcas de maíz como material de soporte

concentración da una primera indicación del comportamiento hidrodinámico del reactor [1]. A partir de esta curva puede obtenerse el Tiempo de Retención Hidráulico (TRH) real. Teniendo en cuenta que las condiciones hidrodinámicas de los sistemas de tratamiento influyen directamente sobre las eficiencias de remoción alcanzadas debido a las características del flujo en la unidad y los períodos de retención del agua en los reactores [2], este estudio se concentró en la evaluación del comportamiento hidrodinámico como herramienta para optimizar un filtro biológico con flujo ascendente.

\section{Metodología}

El estudio del comportamiento hidrodinámico, mediante la técnica estímulo-respuesta consiste en:

- Un reactor compuesto por un tanque de 114 litros, el cual alimenta de agua cruda a tres filtros biológicos tubulares de material PVC con flujo ascendente. Cada uno tiene $100 \mathrm{~mm}$ de diámetro por $1900 \mathrm{~mm}$ de alto, y un volumen de vacío de 8 litros.

- El primer filtro consta de 425 trozos de tusas de maíz con un diámetro de $2.5 \mathrm{~cm}$ y una altura de $2.00 \mathrm{~cm}$. El segundo filtro contiene 600 trozos de tusas de maíz, con un diámetro de 1.5 $\mathrm{cm}$ y una altura también de $2.00 \mathrm{~cm}$. El tercer y último filtro contiene una combinación de las tusas de maíz anteriores trituradas. Respectivamente, cada filtro tiene una densidad de $121 \mathrm{~g} / \mathrm{L} ; 175.61 \mathrm{~g} / \mathrm{L} ; 125 \mathrm{~g} / \mathrm{L}$, con una porosidad de $57 \%$ y superficie específica de $2.52 \mathrm{~m}^{2} / \mathrm{cm}^{3}$.

- El sistema contenía un tanque que almacenaba y distribuía por gravedad un caudal de $125 \mathrm{ml} / \mathrm{min}$ en cada filtro, y en la salida de cada uno de ellos se regulaba su caudal entre de 30$50 \mathrm{ml} / \mathrm{min}$.

- En el punto de entrada del reactor se inyectó $80 \mathrm{ml}$ de un trazador de Cloruro de Sodio (NaCL). Este trazador era medido con un sensor de conductividad Decagon modelo EM50 colocándose en la entrada y salida de cada filtro. Los equipos y reactivos utilizados en el estudio se presentan en la tabla 1, mientras que el diseño conceptual del sistema se esquematiza en la figura 1 .

Tabla 1. Equipos y reactivos utilizados para el estudio hidrodinámico

\begin{tabular}{|l|l|}
\hline \multicolumn{1}{|c|}{ Equipo } & \multicolumn{1}{c|}{ Reactivos } \\
\hline $\begin{array}{l}\text { Reactor conformado por un } \\
\text { tanque y sus tres filtros } \\
\text { biológicos con material de } \\
\text { soporte. }\end{array}$ & $\begin{array}{l}\text { Cloruro de Sodio }(\mathrm{NaCl}) \text { como } \\
\text { trazador }\end{array}$ \\
\cline { 1 - 1 } $\begin{array}{l}\text { Sensor de conductividad } \\
\text { Decagon EM50. }\end{array}$ & \\
\hline
\end{tabular}

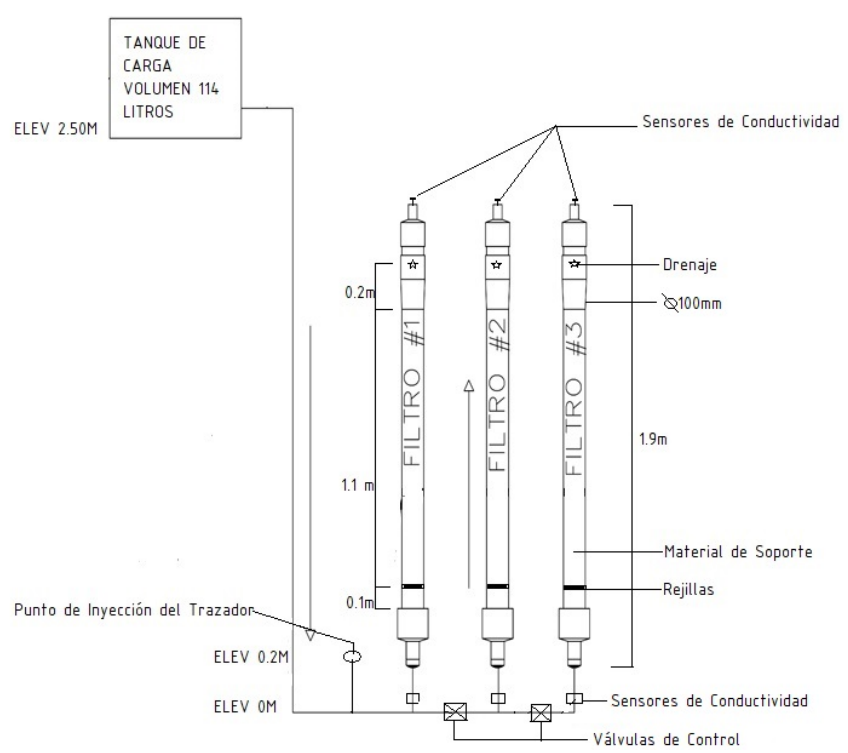

Figura 1. Esquema del filtro biológico de flujo ascendente.

\section{Resultados y discusión}

El análisis de los resultados se realizó a partir de la curva de concentración del trazador vs tiempo. Para determinar el Tiempo de Retención Experimental se utilizó la fórmula 1, la cual se describe en la tabla 2.

$$
\begin{gathered}
t_{e}=\frac{\int_{0}^{\infty} t C(t) d t}{\int_{0}^{\infty} C(t) d t} \\
t_{e}=t_{e}=\frac{\sum_{i=0}^{n}\left[t_{i} C\left(t_{i}\right)+t_{i+1} C\left(t_{i+1}\right)\right]\left(t_{i+1}-t_{i}\right)}{\sum_{i=0}^{n}\left[C\left(t_{i}\right)+C\left(t_{i+1}\right)\right]\left(t_{i+1}-t_{i}\right)}
\end{gathered}
$$

Tabla 2. Significado de la relación de los parámetros de tiempo

\begin{tabular}{|l|l|}
\hline Relación & \multicolumn{1}{|c|}{ Significado (CEPIS, 2004) } \\
\hline$t_{p} / t_{o}$ & $\begin{array}{l}t_{p} / t_{o} \approx 0 \text { (predominio flujo mezcla completa) } \\
\text { si } t_{p} / t_{o} \approx 1 \mathrm{y} \text { ti/to }>0.5 \text { (predominio flujo pistón) }\end{array}$ \\
\hline$t_{i} / t_{o}$ & $\begin{array}{l}t_{i} / t_{o}=0 \text { (flujo mezcla completa) } \\
t_{i} / t_{o}=1 \text { (flujo piston) } \\
t_{i} / t_{o}<0.3 \text { (cortos circuitos) }\end{array}$ \\
\hline$t_{c} / t_{o}$ & $t_{c} / t_{o}=0.693$ (flujo mezcla completa) \\
\hline$t_{b} / t_{o}$ & $\begin{array}{l}t_{b} / t_{o} \approx 2.3 \text { (flujo mezcla completa) } \\
t_{m} / t_{o}\end{array}$ \\
$\begin{array}{l}t_{m} / t_{o}<1 \text { (cortos circuitos y/o zonas muertas) } \\
t_{m} / t_{o}>1 \text { (acumulación indeseada de trazador o error de ensayo) }\end{array}$ \\
\hline $\begin{array}{l}\mathrm{e} \geq 2,3 \text { (flujo mezcla completa) } \\
\mathrm{e}=0 \text { (flujo pistón) }\end{array}$ \\
\hline
\end{tabular}

En donde:

$t_{i}$ : Tiempo inicial desde que se aplica el trazador hasta que aparece en el efluente.

$t_{p}$ : Tiempo modal, correspondiente a la presentación de la máxima concentración 
Mollah (et al): Estudio del comportamiento hidrodinámico de un filtro biológico de flujo ascendente, utilizando mazorcas de maíz como material de soporte

$t_{c}$ : Tiempo de inyección y corrientes de inercia.

$t_{b}$ : Tiempo de inyección y recirculación.

$t_{m}$ : Tiempo mediano, correspondiente al paso del $50 \%$ de la cantidad del trazador.

$t_{0}$ : Tiempo medio de retención o tiempo teórico de retención= V/Q.

$t_{f}$ : Tiempo que transcurre hasta que atraviesa la totalidad del trazador al reactor.

$e$ : Excentricidad de la Curva, en función de la recirculación.

Nota: Todos los tiempos están medidos en unidad de minuto.

Al momento de inyectar el trazador al sistema, dio como resultado gráficas estilo campana para cada uno de los filtros, señalando el recorrido y cada uno de los puntos en donde se pudo establecer los parámetros específicos de tiempo para determinar el tipo del flujo y el comportamiento hidrodinámico, ver tabla 3.

Tabla 3. Análisis de flujos según los parámetros de tiempo

\begin{tabular}{|c|c|c|c|c|}
\hline Parámetro & Filtro 1 & Filtro 2 & Filtro 3 & Observaciones \\
\hline$t_{i}$ & 4 & 15 & 66 & \multirow{6}{*}{$\begin{array}{l}\text { Predominio de } \\
\text { flujo de pistón, } \\
\text { flujo de mezcla } \\
\text { completa, } \\
\text { flujo de pistón, en } \\
\text { cada filtro } \\
\text { respectivamente. }\end{array}$} \\
\hline$t_{p}$ & 32 & 67 & 91 & \\
\hline$t_{c}$ & 56 & 118 & 19 & \\
\hline$t_{b}$ & 80 & 225 & 225 & \\
\hline $\mathrm{t}_{\mathrm{m}}$ & 44 & 117 & 155 & \\
\hline e & 0.48 & 0.72 & 0.66 & \\
\hline
\end{tabular}

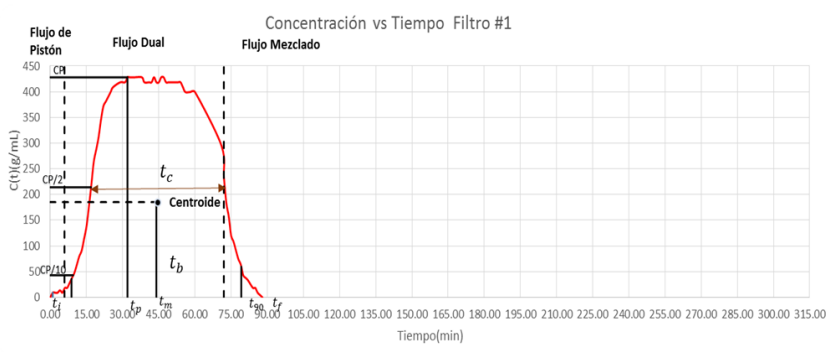

Figura 2. Curva de tendencia de concentración vs tiempo (filtro 1).

En la figura 2 se observa que desde la aplicación del trazador hasta su aparición en el efluente, su tiempo corresponde a $t \_i=4$ min, la fracción de flujo a pistón puede considerarse hasta el primer punto de inflexión de la curva donde cambia de concavidad [4] $(\mathrm{t}=10 \mathrm{~min})$. Entre el punto anterior y el punto de inflexión en la rama descendente de la curva, indicando un tiempo de $\mathrm{t}=60 \mathrm{~min}$, el flujo es considerado dual (pistón y mezclado) y a partir de este tiempo es flujo mezclado (entre 60-88 $\mathrm{min}$ ), ver tabla 4.
Tabla 4. Análisis de la curva de tendencia del trazador del Filtro 1

\begin{tabular}{|l|l|}
\hline \multicolumn{1}{|c|}{ Relación } & \multicolumn{1}{c|}{ Resultado } \\
\hline$t_{p} / t_{o}=32 / 56.69=0.55$ & $\begin{array}{l}\text { Se presentó un corto circuito en el } \\
\text { sistema, produciendo un error en el }\end{array}$ \\
\hline$t_{i} / t_{o}=4 / 57.69=0.07$ & $\begin{array}{l}\text { ensayo del sistema. La excentricidad } \\
\text { de la curva, que está en función de la } \\
\text { recirculación confirma la presencia de } \\
\text { ambos flujos: pistón y mezcla } \\
\text { completa. }\end{array}$ \\
\hline$t_{c} / t_{o}=56 / 57.69=0.97$ & \\
\hline$t_{b} / t_{o}=80 / 57.69=1.39$ & \\
\hline$t_{m} / t_{o}=40 / 57.69=0.69$ & $\mathrm{e}=0.48$
\end{tabular}

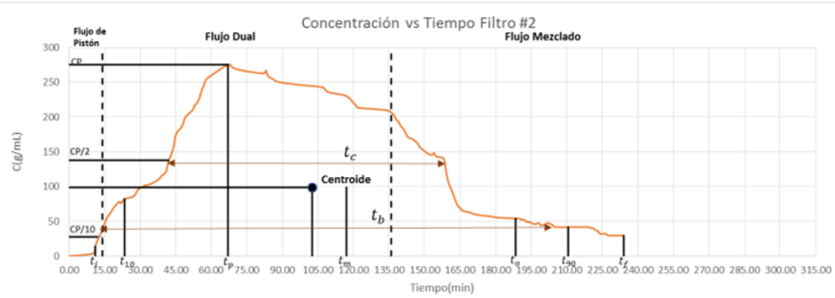

Figura 3. Curva de tendencia de concentración vs tiempo (filtro 2).

En la figura 3 se observa que el tiempo que transcurre desde la inyección del trazador hasta que aparece en el efluente corresponde a $\mathrm{t}_{-} \mathrm{i}=15 \mathrm{~min}$, la fracción de flujo a pistón es considerado hasta el primer punto de inflexión de la curva donde cambia de concavidad [4] $(\mathrm{t}=15 \mathrm{~min})$, ver resultados en la tabla 5. Entre el punto anterior y el punto de inflexión en donde representa el descenso de la curva, corresponde a $t=$ $103 \mathrm{~min}$, el flujo es considerado dual (pistón y mezclado) y a partir de este tiempo nos indica un flujo mezclado (entre $103234 \mathrm{~min}$ ).

Tabla 5. Análisis de la curva de tendencia del trazador del Filtro 2

\begin{tabular}{|c|c|}
\hline Relación & Resultado \\
\hline$t_{p} / t_{o}=67 / 188.37=0.37$ & \multirow{6}{*}{$\begin{array}{l}\text { Se presentaron casos de cortos } \\
\text { circuitos dando variaciones de flujo } \\
\text { de pistón a flujo de mezcla completa } \\
\text { y viceversa con procesos de difusión } \\
\text { debidos a corrientes de inercia. }\end{array}$} \\
\hline$t_{i} / t_{o}=15 / 188.37=0.08$ & \\
\hline$t_{c} / t_{o}=118 / 188.37=0.62$ & \\
\hline$t_{b} / t_{o}=225 / 188.4=1.19$ & \\
\hline$t_{m} / t_{o}=117 / 188.4=0.62$ & \\
\hline $\mathrm{e}=0.72$ & \\
\hline
\end{tabular}

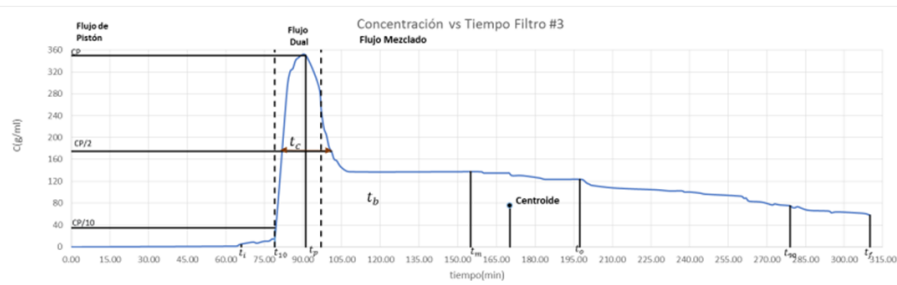

Figura 4. Curva de tendencia de concentración vs tiempo (filtro 3).

En la figura 4 se observa que el tiempo que transcurre desde la inyección del trazador hasta que aparece en el efluente 
corresponde a t_i $=66$ min, la fracción de flujo a pistón puede considerarse hasta el primer punto de inflexión de la curva donde cambia de concavidad [4] $(\mathrm{t}=79 \mathrm{~min})$. Entre el punto anterior y el punto de inflexión en la rama descendente de la curva, que corresponde a $\mathrm{t}=108 \mathrm{~min}$, el flujo es considerado dual (pistón y mezclado) y a partir de este tiempo el flujo predominante es mezclado (entre 108-997 min), ver tabla 6.

Tabla 6. Análisis de la curva de tendencia del trazador del Filtro 3

\begin{tabular}{|c|c|}
\hline Relación & Resultado \\
\hline$t_{p} / t_{o}=91 / 170.26=0.46$ & \multirow{6}{*}{$\begin{array}{l}\text { Dio un indicio de Mezcla } \\
\text { Completa, presentando luego un } \\
\text { corto circuito y una variación de } \\
\text { flujo donde terminó el recorrido } \\
\text { como Flujo de Pistón. }\end{array}$} \\
\hline$t_{i} / t_{o}=66 / 170.26=0.39$ & \\
\hline$t_{c} / t_{o}=19 / 170.26=0.11$ & \\
\hline$t_{b} / t_{o}=225 / 170.26=1.32$ & \\
\hline$t_{m} / t_{o}=155 / 170.26=0.9$ & \\
\hline$e=0.66$ & \\
\hline
\end{tabular}

El tiempo de retención hidráulico (TRH) del sistema es la variación que nos permite conocer el tiempo en que el trazador hace su recorrido por el filtro biológico y pasando así por Volumen Real o espacios vacíos entre las tusas, en tabla 7 se muestra el THR para los 3 filtros.

Tabla 7. Análisis del TRH en cada filtro biológico

\begin{tabular}{|l|l|l|l|}
\hline TRH & Filtro 1 & Filtro 2 & Filtro 3 \\
\hline Experimental & 44.34 & 102.56 & 170.26 \\
\hline Teórico & 58.34 & 189.93 & 197.98 \\
\hline
\end{tabular}

Siendo estos valores menores que el TRH teórico, de acuerdo con [5] el desplazamiento de la curva $C(t)$ hacia la izquierda del TRH teórico es un comportamiento típico de un reactor con presencia de espacios muertos. Comparando ambos valores de TRH, se puede estimar la fracción de zonas muertas $\left(f_{m}\right)$ a partir de la diferencia entre la unidad y la relación TRHreal/TRHteórico, teniendo entre los tres filtros los siguientes resultados:

Filtro 1: 0.76 espacios muertos

Filtro 2: 0.54 espacios muertos

Filtro 3: 0.86 espacios muertos

Tabla 8. Análisis de Volumen Real

\begin{tabular}{|l|c|c|c|}
\multicolumn{5}{c}{ Tabla 8. Análisis de Volumen Real } \\
\begin{tabular}{|l|c|c|c|}
\hline \multicolumn{1}{|c|}{ Elemento } & Filtro 1 & Filtro 2 & Filtro 3 \\
\hline $\begin{array}{l}\text { Volumen de } \\
\text { vacío }\end{array}$ & $8 \mathrm{~L}$ & $8 \mathrm{~L}$ & $8 \mathrm{~L}$ \\
\hline $\begin{array}{l}\text { Volumen de } \\
\text { Tusas }\end{array}$ & $4.17 \mathrm{~L}$ & $2.12 \mathrm{~L}$ & $0.1013 \mathrm{~L}$ \\
\hline $\begin{array}{l}\text { Volumen Real } \\
\text { teórico }\end{array}$ & $3.83 \mathrm{~L}$ & $5.88 \mathrm{~L}$ & $7.89 \mathrm{~L}$ \\
\hline TRH exp & 44.34 & 102.56 & 170.26 \\
\hline $\begin{array}{l}\text { Caudal } \\
\text { promedio }\end{array}$ & 41.6 & 42.5 & 40.5 \\
\hline $\begin{array}{l}\text { Volumen Real } \\
\text { Exp }\end{array}$ & $1.84 \mathrm{~L}$ & $4.45 \mathrm{~L}$ & $6.89 \mathrm{~L}$ \\
\hline
\end{tabular}
\end{tabular}

Vol. 4 - N. ${ }^{\circ} \mathrm{I} \cdot$ Junio $\cdot 2018$
Por medio de las características y dimensiones de las tusas de maíz se pudo calcular el volumen que ocupa, que, en conjunto con el volumen de vacío, se pudo establecer el Volumen Real Teórico para cada uno de los filtros. Obtuvimos un Volumen Real Experimental basándonos en la relación del caudal promedio regulado en el ensayo y el TRH resultante de la prueba del trazador, ver tabla 8 .

Estos volúmenes nos dieron como resultado valores diferentes, ya que al momento de la aplicación del trazador las tusas de maíz ya contaban con un tiempo de degradación mayor al momento en que se construyó el sistema.

\section{Conclusiones}

Actualmente estos ensayos son utilizados principalmente para determinar los tiempos reales de retención y sus principales características concomitantes: tipos de flujo, espacios muertos y cortocircuitos hidráulicos en unidades de tratamiento como mezcladores rápidos, floculadores, sedimentadores, así como en modelos de reactores en etapa de diseño para conocer su comportamiento hidráulico y deficiencias en forma previa a su construcción, lo cual es de enorme utilidad práctica.

Es conveniente, además, escoger como trazador aquella sustancia que no reaccione con los compuestos que existen en el agua. Por tanto, la concentración total que se determine a la salida será sensiblemente igual a la que se aplique a la entrada.

Los diferentes Flujos como de Pistón y de Mezcla Completa, que predominó en el funcionamiento del sistema en los diferentes filtros bilógicos durante el ensayo, se deben al corto circuito que son causados principalmente a las variaciones de concentración del trazador en las pruebas realizadas.

\section{AGRADECIMIENTO}

Nuestro mentor el Profesor Erick Vallester, es digno de orgullo y admiración, pues tiene una larga carrera de entrega a su trabajo. Él con su espíritu altruista, dedicación y estímulo con este proyecto nos ha enseñado la importancia del recurso natural, en este caso el agua, por medio de trazadores, para el estudio de su comportamiento hidrodinámico aplicado en un filtro biológico, siendo este un proyecto interesante y económico para la población, ya que se puede utilizar en beneficio de todos. 


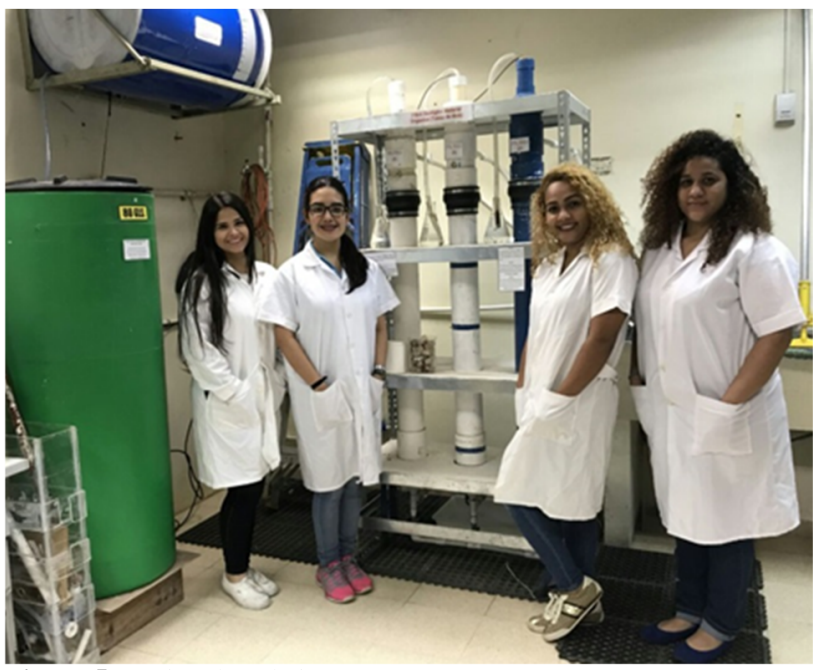

Figura 5. Equipo de trabajo.

\section{REFERENCIAS}

[1] G. Giácoman, L. I. Rejón, S. R. Aguilar. "Evaluación de las características hidráulicas de un reactor de lecho fluidificado para el tratamiento aerobio de las aguas residuales". México. XV Congreso Nacional de Ingeniería Sanitaria y Ciencias Ambientales. 2006.

[2] J. Rodríguez, P. Torres. "Optimización del diseño, manejo, operación y control de un filtro anaerobio a escala piloto para la depuración de las aguas residuales del proceso de extracción de almidón de yuca de la región del cauca. Definición de parámetros de diseño para escalamiento". Proyecto de investigación - Infor- me final. Cali, Colombia. PRONATTA, Universidad del Valle, COPRAUCA. 2002. pp. $10-45$.

[3] G. Galvis. "Consideraciones sobre la aplicación del modelo simplificado de Wolf y Resnick". Barranquilla. Colombia. XXVII Congreso Nacional de Ingeniería Sanitaria y Ambiental. 1984.

[4] D. Hincapié. "Cinética e hidrodinámica aplicadas, en condiciones ambientales locales, a humedales de flujo superficial a escala piloto". Pereira, Colombia, Universidad Tecnológica de Pereira, mayo 2017.

[5] L. Marín "Determinación Experimental de la Distribución de Tiempos de Residencia en un Estanque Agitado con Pulpa". Julio 2007. 